\title{
Théologiques
}

\section{Poétique et imaginaire de la ville contemporaine}

\section{Laurette Wittner et Daniel Welzer-Lang}

Volume 3, numéro 1, mars 1995

Symbolique urbaine et foi chrétienne

URI : https://id.erudit.org/iderudit/602413ar

DOI : https://doi.org/10.7202/602413ar

Aller au sommaire du numéro

Éditeur(s)

Faculté de théologie de l'Université de Montréal

ISSN

1188-7109 (imprimé)

1492-1413 (numérique)

Découvrir la revue

Citer cet article

Wittner, L. \& Welzer-Lang, D. (1995). Poétique et imaginaire de la ville contemporaine. Théologiques, 3(1), 27-41. https://doi.org/10.7202/602413ar

\section{Résumé de l'article}

La complexité urbaine questionne notre intelligence des sociétés. La ville secrète un imaginaire dense et vivant fait de son histoire, des espaces et de leurs représentations, dont seul le récit rend partiellement compte.

L'espace n'est pas polysémique : il inscrit les valeurs dominantes.

L'architecture et l'urbanisme connotent une conception du monde, que l'acte d'habiter travaille, use et parfois modifie, mais cet acte lui-même est appauvri par l'idéologie.

La ville est un empilement fractal de territoires dans lesquels les hommes se déplacent et se côtoient sans forcément se trouver. Le social se structure dans ce qui est l'épaisseur des univers urbains, l'imaginaire vécu, l'espace représenté. Certaines formes urbaines sont devenues le bouc émissaire du mal de vivre. Leurs habitants, objets et victimes de la stigmatisation des lieux, dressent pour se (en) sortir des stratégies de départ réel ou symbolique. L'action sur l'espace, entreprise au nom du bien, se révèle une action de domination sur les hommes. L'approche poétique des espaces permet de retrouver l'humanité de l'autre.

Lisbonne ma ville mon poème de chaque semaine (chanson portugaise) 


\section{Poétique et imaginaire de la ville contemporaine}

Laurette WITTNER enseignante chercheure au Laboratoire de sciences humaines et sociales de l'École nationale des travaux publics de l'État

Daniel WELZER-LANG enseignant chercheur au CREA Université Lumière Lyon II

\section{RÉSUMÉ}

La complexité urbaine questionne notre intelligence des sociétés. La ville secrète un imaginaire dense et vivant fait de son histoire, des espaces et de leurs représentations, dont seul le récit rend partiellement compte.

L'espace n'est pas polysémique: il inscrit les valeurs dominantes. L'architecture et l'urbanisme connotent une conception du monde, que l'acte d'habiter travaille, use et parfois modifie, mais cet acte lui-même est appauvri par l'idéologie.

La ville est un empilement fractal de territoires dans lesquels les hommes se déplacent et se côtoient sans forcément se trouver. Le social se structure dans ce qui est l'épaisseur des univers urbains, l'imaginaire vécu, l'espace représenté. Certaines formes urbaines sont devenues le bouc émissaire du mal de vivre. Leurs habitants, objets et victimes de la stigmatisation des lieux, dressent pour se (en)sortir des stratégies de départ réel ou symbolique. L'action sur l'espace, entreprise au nom du bien, se révèle une action de domination sur les hommes. L'approche poétique des espaces permet de retrouver l'humanité de l'autre.

Lisbonne ma ville mon poème de chaque semaine 


\section{La mise en récit de la ville}

Ville : la vivre et la raconter, ou plutôt, dans l'autre sens, la raconter et la vivre parce que l'homme est avant tout autre chose, un être qui raconte, et qui, pour organiser sa pensée ou ses émotions, doit les mettre en parole.

Ceux qui se sont attaqués à la description de la ville, en scientifiques et non en poètes, ont utilisé le récit. Récit régi par le modèle de

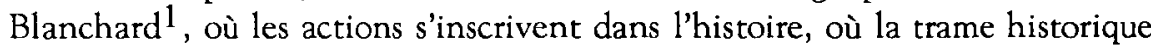
donne un caractère inéluctable aux événements et où le héros est le bâti; ou récit à la manière de Levainville ${ }^{2}$, plus proche de l'épopée, où la ville apparaît en toile de fond des affaires d'hommes ou de classes, et où le héros est l'homme... récit planificateur, aménagiste ou politique comme le veut la fin du siècle, où le héros est climat ou idéologie, mais en tout cas récit. C'est le récit comme forme littéraire, qui a construit l'expression de la géographie, de l'histoire et des études urbaines.

Parce qu'elle a un rythme et des procédés rhétoriques qui lui sont propres, la mise en récit a été rejetée, comme la littérature a pu l'être des champs scientifiques à l'époque où le positivisme connaissait un succès - diffus mais certain - en sciences sociales. Elle a été dévalorisée par le modernisme, qui la méprisait ouvertement et qui l'opposait au savoir, l'accusant de trop de subjectivité et la jugeant insuffisamment explicative. Bannie, la mise en récit a donné place à des tentatives de description, copiées des sciences de la nature. Toute connaissance devait se plier au nouveau modèle qui se voulait garant de l'objectivité scientifique. Seule la philosophie pouvait encore, du haut de ses titres incontestés de noblesse, se permettre la poésie. Mais sans le récit, le discours sur la ville perdait de son pouvoir d'expression. Le récit conférait du sens au discours sur l'homme et la société en ordonnant la réalité; il était le seul à pouvoir rendre compte de la subjectivité sous-jacente aux questions que pose la ville.

La ville échappe en effer à tour discours, et reste inexplicable, gouffre des disciplines, toutes insuffisantes à appréhender la complexité de cette création humaine par excellence, de cet abri démesuré, lieu de l'Homme.

1 Nous faisons référence aux monographies de Grenoble et de Annecy par Raoul BlAnCHARD : Annecy : Essai de géographie urbaine. Annecy, Société des amis du vieil Annecy, 1958; Grenoble: Étude de géographie urbaine. Paris, A. Colin, 1911.

2 J. LeVAINVILLE, Rouen pendant la guerre. Paris, PUF, 1926. 


\section{La ville paradoxale}

Au Moyen Âge, la ville était lieu de tous les maux, de tous les dangers. Lieu laïque et nouveau, où tout était possible et où le droit chemin n'était encore tracé ni par le temps ni par l'expérience, elle ne pouvait qu'être inquiétante. Elle perdure aujourd'hui en cette voie : lieu trouble, de conflits intimes, de contradictions et paradoxes, de complexité et simplicité, d'échanges et indépendance, de permanence et changement, de peur et violence, mais aussi de solidarité et d'appui... La raison première de la ville est que l'homme est incapable de ( sur) vivre seul.

Dans ses franges connotatives, "Cité " décline citoyenneté, civilité. Lieu voulu et recherché, symbole de la modernité au point que le mot "paysan" devient péjoratif en tant qu'étape arriérée du monde, revers d'une même médaille qui indiquerait le mauvais choix. Et pourtant la ville, si convoitée soit-elle, est aussi le lieu de propagation de l'épidémie, de l'insécurité, du vandalisme, des drogues, des décharges, car elle est fruit de ce qui existe comme artifice dans la technique, dans le dressage du temps et des choses, dans la force et l'énergie nécessaires pour que la rivière coule sage et potable aux robinets. La ville est l'écran qui protège l'homme de la nature et lui permet de la rêver. Elle est le lieu de la contradiction née du rejet amoureux d'une nécessité : celle de l'inévitable et de l'évident.

Lourde comme un rêve, la ville prend, comme les rêves, une source dans les représentations du passé et une autre dans celles du présent immédiat. La ville est humaine, abri fait de travaux et tensions, territoire d'enjeux, luttes, pouvoirs. Création, la ville est surtout créative, carrefour du passé, du présent et du futur. La ville est tourbillon de vie, de travail, de mort. Mais qu'est au juste la ville?

\section{La ville illimitée}

Inutile de chercher ses limites sur la carte: la ville se dépasse ellemême. Le rural est contaminé par l'urbain qui lui infuse ses références. L'espace n'existe plus au naturel, les campagnes sont le fruit du labeur agricole, l'inaccessible se fait paysage. Les seuls espaces sauvages, le seul refuge qui reste à la nature sont les friches de ville. Dans les lieux abandonnés et laissés pour compte, dans les franges de l'urbain, dans ces espaces dévolus à personne et abandonnés au temps, la nature reprend ses droits et certaines espèces animales et végétales, s'y trouvant à abri de l'homme, survivent à son insu. L'homme n'est absent que de ce qu'il délaisse. 
Comment cartographier une ville, radiographier ce par quoi l'urbain se métabolise en Cité? Comment établir la frontière, tracer, en dehors de l'administratif, un trait précis de séparation entre centre et banlieue, bon et mauvais? Des frontières existent pourtant, parfois infranchissables. Des hommes gravitent sans jamais se toucher dans des espaces topologiques distincts. Le balayeur de rue, malgré sa nouvelle désignation de "technicien de surface ", n'est qu'une partie de la machinerie du nettoyage, un prolongement du balai, pour ceux qui le croisent à l'œuvre. Dans les gares, il est désormais possible de choisir entre "la main derrière le guichet » ou l'écran tactile. Le choix entre l'un et l'autre est le plus souvent dû à une estimation de la durée de service escomptée. Derrière le guichet, l'homme est machine. Dans le métro, en heures de pointe l'autre-en-face, qui serre, qui prend une place égale à sa surface, n'est pas de la même nature que la machine utilitaire du guichet : comme les pierres sur le chemin, il est entrave, empêchement, difficulté à surmonter. Il ne sort pas de son anonymat, mais seulement de son invisibilité. Il existe, mais pas en tant qu'homme: comme un obstacle, comme une entrave. Le contact avec lui tient de la gêne et non de la communication. Les territoires s'intersectent sans que l'humanité dans l'autre soit perceptible. Seul un accident fait reconnaître, par identification, l'homme dans l'inconnu. Seule la projection réduit les espaces topologiques à leur dimension commune et les rend communicables. Et pourtant, la ville se veut territoire commun, lieu public. La ville se veut Cité. Par la force que donne au sens la proximité phonématique, elle se veut aussi le lieu de la citoyenneté.

La ville serait-elle, comme le voulait Lefebvre $^{3}$, la projection des rapports sociaux au sol? Dans l'affirmative, cette projection pourrait-elle n'être, de manière linéaire et presque univoque, que celle du pouvoir et de l'imaginaire du bâtisseur, les armes étant inégales entre la normalisation et la révolte? Elle serait en ce sens la mise en espace des valeurs que la classe dominante voudrait imposer ou faire partager. Sans une vision de la société où certains droits s'habillent volontiers en faveurs et où l'espace peut être social ${ }^{4}$, il serait difficile de concevoir qu'une classe sociale loge -nécessairement- l'autre. Les logés subissent la construction et les valeurs

3 Henri LefEBVRE, «La vie sociale dans la ville », dans Du rural à l'urbain, Paris, Anthropos, 1962.

4 Considérons une aberration grammaticale, et pas comme une évidence historique, dans le couplet substantif - adjectif " logement social ", la qualification de social appliquée à un espace, et immédiatement la vision des choses bascule en questions d'une certaine pertinence. 
afférentes, la réinterprètent, s'auto-interprètent. Faute de pouvoir ériger, faute de pouvoir modeler à leur image, ils possèdent des outils de déconstruction. Les représentations dominantes se teintent volontiers d'une universalisation usurpée mais crédible. Ainsi on hiérarchise les cultures, les hommes et les lieux.

\section{La ville aux multiples représentations}

Parallèlement aux représentations de l'espace existent aussi des espaces de représentation. Dans leur entrecroisement, l'autre devient sujet : je n'atteindrai mon propre vécu que par l'écho des valeurs reçues de lui dans l'échange. La représentation de soi devient communication. Or, pour communiquer, il est nécessaire de disposer d'un système de règles qui crée de l'intelligibilité. La création de règles comporte un système de valeurs, avec tout ce qu'il peut impliquer de manipulation de l'autre. Derrière les projets, et dans le maçonnement des villes, se discerne une visée, secrète ou inconsciente peut-être, mais bien réelle. L'architecture connote une conception du monde; l'acte d'habiter la travaille, l'use, la modifie parfois, mais cet acte lui-même est appauvri par l'idéologie: finalement, les représentations font partie de la panoplie d'outils de sauvegarde, de stabilisation et de conservation de ce qui est donné au départ comme valeur. Ainsi, lors d'une enquête, un Marocain, las d'être un "immigré » avec le poids négatif que ce mot comporte, finissair par dire -et même croire- que son immeuble était très bien car il n'y avait pas d'étrangers $^{5}$ : se nier en tant qu'étranger était la seule façon à sa portée d'accéder à une représentation positive de soi-même.

Les représentations sont la toile de fond sur laquelle se déploient les actions sociales et sur laquelle se dessine la ville. Tel quartier est vieux mais charmant. Tel autre dangereux. Cette ville est une ville-dortoir même si, objectivement, elle a plus d'emplois que d'habitants. Ce grand ensemble est en béton, même s'il est le champion des espaces verts selon les cadastres. Ces tours que voilà doivent être démolies même si l'on a un besoin urgent de logements, et comme les boucs émissaires elles incarnent le mal de vivre. Le bien et le beau vont ensemble, l'éthique et l'esthétique se donnent la main depuis longtemps. Les beaux quartiers sont riches et inversement. Propre et ordonné, le beau sent la lavande, et correspond aux canons des valeurs culturellement reconnues. L'ensemble éthico-esthétique touche à l'organisation du système social. Qui oserait avouer qu'il aime le

5 Laurette WITTNER, Culture maghrébine et villes de conception occidentale. Thèse de doctorat en urbanisme, 1989 
bruit, la pollution, les ombres chinoises que le soleil couchant dessine sur les décharges? Qui oserait même le penser? Des excentriques ou des artistes, ceux qui par révolte ou par croyance inébranlable dans la sensation comme donnée peuvent se démarquer de la valeur reconnue et s'écarter de son droit chemin. Voyons-en un exemple.

Le bruit, c'est bien connu, est un des maux urbains du siècle. Il faut protéger les logements du bruit. Lors d'un travail sur le logement des personnes âgées, tous nos enquêtés se plaignaient du bruit ambiant. Ils étaient sans cesse dérangés par les voisins, par la rue... Tout était cohérent à un détail près : la plupart des enquêtés étaient malentendants. Le bruit qui les dérangeait n'était pas celui contre lequel s'acharnaient les constructeurs, mesurable en décibels. "Bruit" était le mot intelligible pour dire un mal être diffus et beaucoup plus complexe, inexprimable celui-ci. Quand il s'agit du bruit, tout le monde se met instantanément d'accord : la souffrance est compréhensible, les victimes méritent la complaisance et peuvent espérer que constructeurs et gestionnaires s'occupent d'eux. Cependant, dans le cas étudié, le mal venait surtout de la position sociale des personnes âgées. Elles n'avaient plus de rôle social à jouer et leur logement, indépendamment de sa forme ou localisation, était inconfortable comme peut l'être la salle d'attente de la mort. Les acteurs en présence se sont immédiatement attaqués au bruit, objet de consensus et de plainte. Les logements des personnes âgées concernées, dans une nouvelle construction, étaient bien insonorisés, donnaient sur un parc et obéissaient à la demande des habitants et à tous les stéréotypes. L'ennui et le mal de vivre se sont-ils estompés pour autant? Les enquêtés ont fini par exprimer leur regret de ne plus voir, de leur fenêtre, le mouvement de la rue. Le parc, si demandé, était trop peu changeant pour garder leur attention et la vue de ce lieu calme et aménagé leur faisait penser au cimetière ${ }^{6}$.

Voilà en quoi réside la force des valeurs dominantes: leur intériorisation. L'intériorisation peut être si forte qu'elle est acceptée sans restriction par adhérence de l'esprit. Nous ne pouvons évidemment pas penser ou agir sans direction ou modèle, ce qui oblige à une adhésion de l'esprit. Le danger est celui d'une adhérence inconsciente, qui ne s'interroge donc pas sur elle-même. Universalisant les dominants, elle peut aller jusqu'à la négation d'une autre évidence : le soi-même, l'être soi.

6 Ce qui est une conséquence, un effet pervers récent des cimetières paysagers : ce cimetière/parc finit par associer les parcs aux cimetières. 
De quel espace polysémique se doter, qui puisse accueillir des valeurs et codes différents et permettre l'épanouissement de différentes cultures?

La ville n'est pas seulement l'espace où se dérouleraient linéairement des pratiques et des représentations, un lieu qui comporterait, pour ainsi dire, l'action et le rêve. Il y a dans la ville un empilement des représentations: les territoires se croisent, se superposent, et sont la scène de rapports de conflit ou de pouvoir; ou au contraire, par superposition topologique, ils se traversent et ne se touchent pas. La ville est un espace multiforme. Demandez à un habitant un dessin très précis de sa ville: il commencera à partir de sa maison, comme on commence à voir le monde à partir de soi. De sa maison partiront, en étoile, les axes qu'il emprunte et feront son quartier, sa ville, ce qu'il connaît, ce qu'il vit, et qui ne correspond ni au quartier ni à la ville de son voisin. Les lieux où il ne va pas, les rues non empruntées, sont une masse inconnue et plus ou moins difforme ou plutôt a-forme. Du moins leur forme dépend-elle de la connaissance qu'il peut avoir des cartes, informations, légendes, mais elle n'a pas la structure du vécu. Sur les axes empruntés il connaîtra plus ou moins bien les objets qui les dessinent, selon le mode de transport, l'intensité du passage, le temps disponible et toutes ces choses qui façonnent le regard. Il connaîtra les façades des rues familières au point de pouvoir dire au premier coup d'œil, dans un lieu connu, où il est. Mais la connaissance n'est pas uniforme parmi ces façades reconnues. Même dans l'enfilade de vitrines d'une rue commerçante, il connaît plus les unes que les autres. Les magasins qu'il fréquente ( ou, au contraire, qu'il convoite, qui lui font envie mais sont en dehors de ses besoins ou moyens), sont les plus familiers. Intercalés entre eux, les autres ne sont pas remarquables et sont fréquemment oubliés. La mémoire est sélective et ce qui n'accroche pas l'esprit peut facilement s'invisibiliser pour l'œil. Un ami de notre habitant peut lui "faire découvrir " un restaurant devant lequel il passait pourtant tous les jours car il y a des zones d'ombre dans les chemins connus. Ainsi, la ville, le quartier, ou même la rue parcourue sera différente pour chacun selon ses trajets, ses goûts, son histoire. Mais elle est pourtant, sinon la même ville, du moins composée objectivement du même bâti.

Ces multiples étoiles de lieux traversés et connus, de chemins et d'objectifs qui constituent la ville, ne sont pas seulement parcourus et fréquentés : ils sont aussi imaginés. Les représentations sont à leur tour des constellations de trajets et de lieux. Point n'est besoin de connaître les lieux pour former son opinion. Kant, qui ne quittait jamais sa ville, a pu décrire et disserter sur un pont anglais aussi bien que s'il l'avait croisé toute sa vie, voire mieux. La connaissance peut venir par d'autres voies que 
l'expérience. L'imaginaire peut être informé par le collectif, par la culture qui est appropriation des expériences d'autrui, ou par des sources qui, se voulant "sûres et certaines" (comme la rumeur), déversent les légendes, tissent la mythologie urbaine, nourrissent les récits.

\section{La ville, reflet du social}

Construite de ses pierres et de ses légendes, la ville se bâtit du social. Les hommes vivent autant dans les lieux que dans la réputation des lieux. La violence invisible double et propage la violence visible. Il faut se défendre non de ce qui est, mais de ce qui peut arriver. L'ennemi invisible est comme l'angoisse, vulnérant, quoique impalpable et sans objet déterminé. Les formes urbaines servent parfois de paravent aux questions qui, posées, pourraient ébranler le modèle qui supporte l'ordre social. À qui la faute si les hommes ne naissent pas tous avec les mêmes possibilités d'exercice de leurs égalités? La différence s'imprime dans la ville et cette marque défigure une société qui se veut égalitaire, en invalidant ses prétentions. Le bâti expose à ciel ouvert ce que le discours tend parfois à lisser ou à gommer; il oblige à des explications et théorisations contraignantes.

Nous pourrions trouver une beauté dans les banlieues ${ }^{7} \mathrm{~s}^{\prime} i l$ nous était possible de les voir sans l'ensemble de connotations négatives qui les revêt d'une couche opaque et infrangible de laideur. Mais causes et conséquences ne sont pas de l'ordre de l'esthétique, du plaisir de l'œil. La critique de l'architecture des habitations est plus épaisse, plus lourde de conséquences, plus multidimensionnelle que celle de l'œuvre d'art. L'espace jugé est habité. Dans l'ensemble des langues indo-européennes le verbe habiter participe des racines d'avoir, être, construire et vivre. Le logement est une deuxième peau qui colle à l'habitant et l'identifie, au regard des autres, à un groupe, à un (stéréo )type; ceci même s'il peut se démarquer à son propre regard, par stratégie ou par projet. Les mots ne permettent-ils ou ne menacent-ils pas de faire confondre les actions sur les hommes et celles sur le bâti? Réhabilitation provient du langage juridique avant d'être utilisé pour les constructions. Réhabiliter signifie selon le dictionnaire ${ }^{8}$, "rétablir dans un état, dans des droits des privilèges perdus; rendre à un condamné ses droits perdus et l'estime publique en reconnaissant son innocence ». Le mot ne s'est appliqué au bâti que très

7 Il s'agit ici des banlieues troubles de certaines villes françaises et non des banlieues nord-américaines en principe "proprettes» (NDLR).

8 Le Robert. 
récemment : c'est un article du journal Le Monde du 2 novembre 1969 qui parle le premier de "réhabiliter un paysage urbain et industriel dégradé ». Dans l'obscure épaisseur des mots comment ne pas réhabiliter les hommes avec les logements?

Suivons de près quelques habitants d'un lieu qui, ayant perdu l'estime publique, doit être réhabilité.

"Tout le monde sait " par la force de la réputation, que "dans ces quartiers là » tout est possible, et surtout le pire. Cette image qui colle à la peau des lieux abaisse d'emblée le seuil du «normal " et du tolérable, pour de nombreux intervenants, bien au-dessous des critères d'usage. Ici, il en faudrait plus qu'ailleurs pour faire un "fait divers " : l'événement, pour devenir révoltant, et parvenir au jour, devrait d'abord percer la réputation et la distance, cette distance infinie qui sépare les hommes des hommes quand ils se savent non concernés. Les morts du Rwanda sont un alignement de chiffres, pas d'hommes, le choléra n'épouvante plus nos civilisations hygiéniques, et même si une "peur bleue " figure encore comme expression de notre langage, son origine s'est perdue dans le temps; rien ne rappelle plus le teint bleuté qui caractérise cette maladie. Les faits des banlieues sont ceux d'un "ailleurs" dont certains seraient, en quelque sorte, épargnés. Ainsi une infirmière scolaire peutelle déclarer que "c'était une bonne année, où il n'y a pas eu de problèmes: il n'y a pas eu plus d'incestes et de fugues que d'habitude ». De même, un responsable peut considérer que le lycée n'avait pas " de problèmes de drogues" car les jeunes ont "assez de place pour se droguer en bas des tours, pourquoi voulez-vous qu'ils le fassent ici?", ce qui montre qu'il limitait les "problèmes de drogues" auxquels pouvait être confronté un lycée à la seule utilisation ou manipulation dans l'enceinte de l'établissement; qu'une partie de ses élèves soient utilisateurs de stupéfiants ne le surprend pas, ni ne le concerne ${ }^{9}$. À la chercheure étonnée, il répond en donnant effectivement une des clefs de sa vision des choses: "Pourquoi semblez-vous scandalisée de ma façon de parler de ces problèmes? Mais ma brave dame, où pensez-vous être? Ici, c'est les Minguettes ${ }^{10}$."

9 Propos recueillis lors d'une recherche opérationnelle pour la restructuration d'un quartier de banlieue en agglomération lyonnaise, commune de Venissieux.

10 Minguettes : ensemble de tours d'habitation (HLM) en banlieue de Lyon dont certaines, à peine âgées d'une trentaine d'années, ont été détnolies en 1994 pour insalubrité. (NDLR) 
"Ici " est donc un de ces lieux hors conscience, un non-lieu du tragique. Un lieu où la distance symbolique ne permet pas l'identification. Dès lors, par manque de projection sur soi, on se retrouve dans le cas de la maladie oubliée: l'humanité de l'autre est invisible, et avec elle sa souffrance. En traversant des formes urbaines désignées, on passe de monde à monde, de ville à ville. Certains parlent d'exclusion, de relégation. C'est partiellement vrai s'il s'agit de l'accès à la société et à la logique dominante. Pour ces valeurs là, le chemin est effectivement bouché, faute d'une égalité en matière d'opportunités. Mais comment peut-on parler de l'exclusion ${ }^{11}$ d'un être présent, si ce n'est par refus de le voir? Il n'y a ni océan ni rempart entre la société et ce nouveau lieu dit de relégation. La distance n'est pas infranchissable comme le voudraient, en deçà des discours, les mots qui les composent. Les espaces sont reliés par un même réseau viaire, par des moyens de transport, par des flux et des passages, parfois difficiles mais permanents. Les mondes se traversent, se croisent, liés à jamais par la continuité géographique : sur le plan il n'y a parfois qu'une rue à traverser. Derrière la devise égalitaire transparaît la peur de l'inconnu, de l'autre déshumanisé, de "ces gens-là ". Une fois l'angoisse localisée, on sera en sécurité partout ailleurs. Le bouc émissaire est efficace car il part avec la peur. Si le danger vient d'un certain type d'individu ( disons un jeune, à teint mat, visiblement pauvre, mal habillé ) l'ennemi potentiel est considérablement réduit car tous les autres ne sont plus des agresseurs potentiels : la rue devient possible. Si le danger vient des banlieues, il n'est pas ailleurs. Il suffirait alors de réduire les passerelles pour que la ville soit sûre et propre et pour que les jeunes de bonne famille ne croisent pas des dealers. Dans cette logique, une jeune étudiante qui habitait un quartier réputé difficile, s'étant fait agresser en plein centre-ville a déménagé, par peur, de son quartier. Accrue de son expérience, sa peur devenait insupportable et handicapante. La projection de cette peur sur un lieu déterminé, en l'occurrence le quartier où elle habitait et que l'on considérait dangereux, lui laissait le libre usage du reste de la ville. Mais quitter son logement de banlieue n'est pas la solution pertinente pour éviter une agression en centre-ville, et le bouc émissaire qui s'en va avec la peur emporte aussi ce qui pourrait produire des changements véritables: le regard critique de l'homme sur soi-même, sa responsabilité dans le mal du monde.

11 L'exclusion est un terme très intéressant parce qu'en plus de l'action, il a, selon le dictionnaire une référence spatiale incontestable. Action d'exclure en chassant d'un endroit dont il avait précédemment sa place. 


\section{La ville qui colle à la peau}

Changeons d'optique. Les "exclus " eux-mêmes ne s'auto-identifient pas comme tels. Ils circulent dans l'espace qui leur est accordé ${ }^{12}$ et contraignent l'autre à leur présence. Sauf par apprentissage des termes à la mode, ils diront injustice ou inégalité plutôt qu'exclusion. Ils réclameront des droits, des opportunités, des équipements, des services; revendiqueront peut-être de la justice. Leur "exclusion" est un imaginaire qui n'est pas le leur et ne les touche pas. Comme le Marocain qui ne se savait plus l'immigré de son immeuble, ils ignorent qu'ils ne sont pas là, qu'ils ont été portés séparés, disparus dans un bagne urbain et social. Quand ils se déplacent dans la ville, ils ne s'aperçoivent pas de l'infranchissabilité des barrières, et les traversent. Cerres, les accès sont difficiles, à commencer par les transports. Certaines villes trouvent en effet des moyens subtils d'allonger certaines distances, car l'homme circule en temps plutôt qu'en kilomètres, et la distance vécue est donc celle du temps de parcours $^{13}$. Il suffit ainsi d'une politique de transports pour changer la géographie. L'imaginaire devient bâtisseur de distances et la séparation se creuse à coup de temps. S'y ajoutent les contradictions sociales créées par la fuite en avant des strates dominantes qui, pour se démarquer des autres, transmutent inlassablement le luxe en confort nécessaire, ce qui a pour résultat de rendre l'indispensable hors de portée d'une partie de la population. Présents sur la scène urbaine malgré « l'exclusion» dont ils seraient victimes, les dits exclus mettent en place des stratégies de survie au sein d'une société qui n'est pas vraiment faite à leur mesure et qui, à la fois sujet et lieu, s'acharne à leur enlever leur visa.

Pour se laver des taches d'un quartier désigné, la seule solution est d'en sortir: la réputation du lieu traîne et entraîne irrémédiablement l'habitant. On objectera que celui-ci peut parfois, par l'épaisseur insistante de sa présence, se faire reconnaître et être connu, être vu en tant que lui-

12

Beaucoup de jeunes de banlieue, nous avons pu le constater dans nos propres terrains de recherche, réagissent mal aux politiques et aux projets mis en œuvre pour améliorer leur quartier, car ils leur semblent une manière de "dorer les barreaux de la cage où on veut nous enfermer". D'où, au moins dans les termes, le glissement sémantique des politiques de DSQ (Développement Social des Quartiers) en DSV (Developpement Social des Villes).

13 Paris est devenu plus «proche " de Lyon, lors que le TGV a réduit le temps de parcours de quatre à deux heures de voyage. Ce qui est plus accessible (sur la ligne de métro, par exemple), est considéré moins éloigné que ce qui est moins accessible, (rupture de charges, marche à pied) même si la distance en kilomètres est moindre. 
même malgré le brouillard des préjugés. Cependant, il aura alors statur de preuve ou d'exception (n'en faut-il pas une à toute règle, qui la confirme?). Il sera celui qui est "quelqu'un de bien ${ }^{14}$ " mais malgré quelque chose : un «bien " méritant mais douteux, peu fiable en quelque sorte.

L'inertie de la réputation, sa viscosité, sont trop importantes pour permettre à ceux qui la subissent un accès facile aux actes nécessaires à la vie courante. Ainsi, les jeunes composent avec la réputation. "Tout le monde sait " que ceux de banlieue sont, sinon des délinquants, du moins dangereux; au cas où il s'agit de rescapés à la règle, "à coup sûr», ils ont des mauvaises fréquentations qui peuvent un jour leur tourner la tête. Les jeunes finissent en conséquence par donner une autre adresse quand ils cherchent un emploi. L'adresse d'une personne de la famille, d'un ami, de quiconque accepte de prêter sa boîte aux lettres pour l'occasion. Sortir sans sortir, se laver de la faute originelle du lieu, être seulement soimême et responsable uniquement de ses propres défauts, libéré des préjugés face aux conditions d'embauche, voilà la stratégie mise en œuvre. Répondre par un mensonge au mensonge du préjugé. Cette stratégie n'est pas accessible à tous, elle nécessite des connaissances externes, de ne pas être seul. La réputation est un sable mouvant; pour s'en arracher il faut de l'aide extérieure. Mais pour ceux qui réussissent cette évasion, une question reste ouverte : comment peut-on vivre une adresse avilissante, infamante? Comment peut-on se dédoubler et être malgré la dégradation -constatéede son lieu de vie? Parfois le rêve pousse à un objectif précis : le départ. Sortir vraiment, ( s'en sortir dit-on), échapper physiquement à la déshumanisation rampante de l'imaginaire. Et certains des jeunes de banlieue s'échappent. D'autres stratégies sont alors mises en place, allant de l'étude au perfectionnement dans la délinquance. Pour d'autres encore le

14 On se dépare trop souvent avec des propos ouvertement racistes qui font exception des personnes connues : «les arabes, je n'ai rien contre, ils ont le droit de viare mais, vous savez... ce n'est pas bon pour un immeuble car ils font du bruit, ils ont plein de gosses, ils utilisent les paliers, les escaliers... les enfants n'ont pas de discipline, que voulez-vous ? Ceci dit, mon voisin de palier est Marocain, ou Tunisien, je ne sais pas. Ils sont un couple très gentil, on se dit toujours bonjour, quand j'étais malade ils se sont proposés à m'aider pour les courses, c'est gentil, non? Mais eux ils sont différents, ils n'ont pas beaucoup d'enfants, je pense qu'ils ont fait des études, ils doivent avoir une belle position car ils sont très bien habillés, il travaille en costard cravate. Je pense qu'ils ne feront pas des enfants à la douzaine, mais même s'ils en font plusieurs je pense que ça ne sera pas pareil. Il y a une dame, française, au dernier étage, elle a cinq enfants, elle les tient très bien, vous voyez, ce n'est pas le nombre qui compte..." 
projet reste vague, imprécis, comme une envie sans détermination. Ils échappent alors sans s'échapper, dans l'utilisation d'inducteurs chimiques de rêve, dans la recherche de racines improbables, dans la pratique religieuse, dans des moyens à leur portée d'être ailleurs sans quitter les lieux.

Ou alors, d'une manière plus cynique et commerciale, mais aussi plus conformiste, ils vendent leur image telle que la veulent les autres, telle que la réputation l'a modelée. L'ancien loubard devient gardien d'immeuble, de supermarché. Il monnaye sa connaissance du réseau, sa respectabilité, sa force. Un chef de bande aura un meilleur emploi qu'un autre de son groupe: la hiérarchie sociale reste la même. Revers de la médaille, on pourrait dire qu'on ferait alors plus confiance à celui qui été le plus dangereux. Mais la logique n'est pas si linéaire, et l'organisation sociale dispose d'un poids solide reconnaissable et construit.

\section{Prendre la clef des villes}

Il est plus difficile de forcer les barrières sociétales que de traverser les espaces dans la ville. Les trajets amènent en effet à des lieux et l'utilisation du territoire est codée. Toute entrée est privée, toute porte est une question implicite : pouvez-vous vous justifier d'être là? Le Sphinx est le premier contrôleur de frontières internes, le visa d'espace à espace ne tient pas de l'administratif mais de la bonne réponse. Chaque lieu implique des repères, de l'apprentissage et l'utilisation d'un code. Les commerces semblent ouverts à tous mais ils attendent de la consommation. Il faut avoir de l'argent pour les fréquenter, ou s'il s'agit de flâner, avoir moyen ou envie de faire semblant. Les loisirs les plus communs (cinémas, pubs, discothèques) sont payants. Les lieux dits publics ne sont pas à usage de tous. Pour fréquenter une bibliothèque, un centre culturel ou sportif, une maison des jeunes (et nous pouvons décliner ainsi toute la nomenclature du public, du square à l'Hôtel de Ville), il faut du moins l'avoir appris, ou savoir ce qu'on cherche, quoi demander au cerbère souriant de l'accueil. Les hommes n'entrent pas volontiers dans des espaces où ils n'ont rien à faire, où ils ne savent pas se mouvoir. Pour utiliser un lieu il faut déjà le connaître, être initié. La connaissance des espaces s'opère par le réseau d'abord, et procède par analogie dans un second temps : la fréquentation d'un musée ouvre la porte de tous les autres. Le code de manières étant appris, les lieux deviennent familiers, le Sphinx propose toujours la même énigme.

Si la circulation entre les différents espaces est possible, l'aboutissement ne l'est pas. Pour être arrivé il faut répondre à la question implicite, 
la raison d'être là, qui demande justification aux autres et parfois à soimême. Pour les jeunes d'ailleurs, venus de la banlieue au centre et ni vraiment citoyens ni maîtres des lieux, on ne compte pas le nombre de portes fermées, de lieux interdits par délit de mauvaise tête ou de chaussures trop fatiguées. Le "videur " de la discothèque, le patron du bistrot, le gardien du parc ou du bâtiment public jugent à l'habit qui fait le moine ici comme ailleurs : l'avoir et l'être se confondent à se perdre dans les teintes chargées de siècles d'apparence.

Repoussés, les jeunes ne sont pas "exclus " pour autant, par l'effet même de leur présence, surtout en ce qu'elle a de dérangeant. Ils sont dans les lieux interdits dans un transit nomade, une présence de parcours sans point d'accroche, sans un véritable droit de cité. Ils jouent à la fois le rôle du marginal et de l'intégré, de l'exclu et du présent. Selon les situations, et non dans l'absolu, ils seront l'un ou l'autre. La désignation n'est pas un attribut intrinsèque de l'être : son champ de pertinence est un rapport d'adéquation à une situation.

Comme terme, l'exclusion participe du désir et du rêve. Elle tient ses racines de l'envie inavouable de distance, colorée de cette pitié qu'il est loisible aux gens de mettre en œuvre.

Les acteurs sociaux techniques et politiques sont unanimes : il faut casser, réhabiliter, changer. La forme urbaine des barres et des tours est inhumaine et provoque la cassure et le mal de vivre. Un architecte expliquait très sérieusement comment il redonnait la dignité aux gens en rompant les verticales de lumière des barres. Tous les ouvriers ayant à peu près les mêmes horaires, leurs immeubles vus de l'extérieur manifestent des verticales éclairées (dégradantes selon lui ), correspondant à l'heure où les familles sont dans la cuisine, dans le salon, devant le téléviseur... Il suffisait donc de décaler les pièces et donner un rythme aux immeubles pour que la verticalité lumineuse se casse, faisant retrouver la dignité perdue. Il n'est pas une caricature, cet architecte, metteur en scène du décor d'une immense pièce technique dont les habitants sont des figurants; il est à la fois auteur et réceptacle d'un courant de pensée : celui qui alimente les notions que nous venons de parcourir. Il est le cas extrême d'une pensée née de la déshumanisation de l'autre. L'architecte dieu ou roi qui dispose de la dignité des hommes comme si elle lui appartenait, comme si elle dépendait du dessin formé dans le paysage par l'éclairement de leurs ampoules électriques, est le frère jumeau de tous ceux qui croient résoudre des problèmes de société par la seule action sur la forme urbaine. 
Les hommes devraient avoir droit, à chaque époque de leur vie, à des logements qui leur conviennent. Ils devraient pouvoir suivre leur stratégie résidentielle telle qu'eux-mêmes la définissent. Il est possible qu'une grande partie de l'effort fait pour réhabiliter consiste, pour citer l'image si juste d'un jeune de Venissieux, à "redorer les barreaux de la cage " pour laisser certains parqués sans rémission et sans remords dans l'espace qui leur a été accordé.

Il se peut aussi que l'action sur leur espace, entreprise au nom du bien, soit encore une fois une action de domination sur les hommes. On accorde la banlieue à la tonalité de la ville, on la fait rentrer de force comme un objet de seconde classe dans les valeurs dominantes: laide, pauvre, mais au moins propre. On a beau nettoyer à outrance au nom de cette liaison profonde entre l'homme et le lieu, l'être de l'homme dépasse la conceptualisation et l'espace polysémique est parfois en dehors de luimême. Une enquêtée d'un grand ensemble était très satisfaite de l'état de son immeuble avant réhabilitation : la modicité du loyer lui avait permis de s'acheter un appartement sur la Côte d'Azur. Son objectif n'a été possible et réalisable que grâce à une vétusté qui aurait dû, en bonne théorie, y faire obstacle. La saleté de son immeuble a été sa réussite, réussite cachée et invisible à une vision linéaire et sans profondeur. Les espaces s'empilent et les lieux se créent à l'infini dans les creux des lieux. Même le récit est trop rigide pour rendre compte de la densité fractale de la ville. Reste la poésie qui joue en lieu et en forme avec la forme du lieu. 